University of Nebraska - Lincoln

DigitalCommons@University of Nebraska - Lincoln

September 1967

\title{
High-Field Magnetoresistance of Ordered Beta Brass
}

David J. Sellmyer

University of Nebraska-Lincoln, dsellmyer@unl.edu

J. Ahn

Massachusetts Institute of Technology, Cambridge, Massachusetts

J.-P. Jan

National Research Council, Ottawa, Canada

Follow this and additional works at: https://digitalcommons.unl.edu/physicssellmyer

Part of the Physics Commons

Sellmyer, David J.; Ahn, J.; and Jan, J.-P., "High-Field Magnetoresistance of Ordered Beta Brass " (1967). David Sellmyer Publications. 185.

https://digitalcommons.unl.edu/physicssellmyer/185

This Article is brought to you for free and open access by the Research Papers in Physics and Astronomy at DigitalCommons@University of Nebraska - Lincoln. It has been accepted for inclusion in David Sellmyer Publications by an authorized administrator of DigitalCommons@University of Nebraska - Lincoln. 


\title{
High-Field Magnetoresistance of Ordered Beta Brass*
}

\author{
D. J. Sellmyer and J. Ahn $\dagger$ \\ Massachusetts Institute of Technology, Cambridge, Massachusetts \\ AND \\ J.-P. JAN \\ National Research Council, Ottawa, Canada
}

(Received 27 March 1967)

\begin{abstract}
The magnetoresistance of several single-crystal specimens of ordered $\beta$-brass has been investigated in fields up to $150 \mathrm{kG}$. The purity of the samples is sufficiently high $\left(\rho_{295} / \rho_{4.2} \simeq 400\right)$ that the high-field condition is satisfied. The results indicate that ordered $\beta$-brass is an uncompensated metal and that its Fermi surface is multiply connected. Strong open orbits were observed when the field was in $\{110\}$ or $\{100\}$ planes, and this behavior is compatible with the Fermi-surface model predicted by augmented-plane-wave and Green's-function energy-band calculations. Evidence for the existence of higher-order open orbits and two-dimensional regions of open orbits is presented. There are certain aspects of the data which cannot be explained in terms of the theoretical model unless (a) one of the predicted open orbits is eliminated either by magnetic breakdown or by changing some of the model's dimensions, and (b) other open orbits due to magnetic breakdown are taken into account. Various possibilities for open orbits due to magnetic breakdown are considered and their implications are compared with the observed behavior.
\end{abstract}

\section{INTRODUCTION}

$\mathrm{T}$ HE last decade has seen great advances in our understanding of the electronic structure of pure metals. This progress has been due, in large measure, to the success of such Fermi-surface experiments as the de Haas-van Alphen (dHvA) effect and highfield magnetoresistance. These experiments depend upon the electrons executing several cyclotron orbits before being scattered so that low temperatures and rather pure materials are required. In the past several years it has been shown that certain metallic compounds and ordered alloys also can be prepared with a rather high degree of crystalline perfection. This means that the high-field condition mentioned above, $\omega_{c} \tau>1$ where $\omega_{c}$ is the cyclotron frequency and $\tau$ the relaxation time, can be achieved in these metals as well as in elemental metals and, of course, implies that the Fermi surfaces in this class of metals can be investigated experimentally in detail. Indeed, a number of ordered alloys have been investigated with the dHvA effect $^{1-7}$ and with high-field magnetoresistance. ${ }^{8,9}$

\footnotetext{
* The research at MIT was supported by the Advanced Research Projects Agency under contract No. SD-90, through the Center for Materials Science and Engineering.

$\dagger$ IBM Resident Graduate Fellow.

1 A. Beck, J.-P. Jan, W. B. Pearson, and I. M. Templeton, Phil. Mag. 8, 351 (1963).

2 J.-P. Jan, W. B. Pearson, and M. Springford, Can. J. Phys. 42, 2357 (1964).

${ }^{3}$ J.-P. Jan, W. B. Pearson, and M. Spingford, Ninth International Conference on Low-Temperature Physics (Plenum Press, New York, 1965), p. 776.

${ }^{4}$ J.-P. Jan, W. B. Pearson, and Y. Saito, Proc. Roy. Soc. (London) A297, 275 (1967).

${ }^{5}$ G. J. Edwards, M. Springford, W. B. Pearson, and Y. Saito, Proceedings of the Tenth International Conference on Low-Temperature Physics, 1966 (to be published).

${ }^{6}$ J.-P. Jan, W. B. Pearson, Y. Saito, M. Springford, and I. M. Templeton, Phil. Mag. 12, 1271 (1965).

${ }^{7}$ B. W. Veal and J. A. Rayne, Phys. Letters 6, 12 (1963).

8 D. J. Sellmyer and P. A. Schroeder, Phys. Letters 16, 100 (1965).

J. Longo, P. A. Schroeder, and D. J. Sellmyer (to be published).
}

In this paper we report an investigation of the highfield magnetoresistance of ordered $\beta$-brass $\left(\beta^{\prime}\right.$-CuZn). This alloy is particularly interesting because it is the traditional prototype of a set of alloys, the HumeRothery electron compounds, which strongly demonstrate the influence of electron concentration on crystal structure. A detailed knowledge of the electronic structure of these compounds should therefore serve as a starting point for an understanding of the factors which govern phase stability in solids. ${ }^{10}$ In addition, $\beta^{\prime}$-CuZn is one of the few ordered alloys on which there have been done first-principles energy-band calculations; Arlinghaus ${ }^{11}$ using the augmented-plane-wave (APW) method and Johnson and Amar ${ }^{12}$ and Amar et al. ${ }^{13}$ using the Green's-function method. Jan et al. ${ }^{4}$ have measured the dHvA effect in $\beta^{\prime}$-CuZn, $\beta^{\prime}$-AgZn, and $\beta^{\prime}$-PdIn, all of which have the cubic $\mathrm{CsCl}$ structure. The measured cross-sectional areas are in semiquantitative agreement with the calculations for $\beta^{\prime}-\mathrm{CuZn}$ and $\beta^{\prime}-\mathrm{AgZn}$ although several of the predicted orbits have not been observed; for AgZn and PdIn, there exists a set of dHvA frequencies which cannot be correlated with the predicted Fermi-surface models. The present work was undertaken, therefore, to provide a further test of the theoretical calculations on $\beta^{\prime}$-CuZn.

\section{THEORY}

\section{A. Rules for Compensation in Ordered Alloys}

Let $V_{e}\left(V_{h}\right)$ be the electron (hole) volume of the Fermi surface in $\mathbf{k}$ space, $\Omega$ be the volume of the primitive cell, and $n_{e}\left(n_{h}\right)$ be the number of electron (hole)

\footnotetext{
${ }^{10}$ See P. W. Anderson, Concepts in Solids (W. A. Benjamin Inc., New York, 1963), Chaps. 1 and 2, for a lucid discussion of the band theoretical versus the quantum-chemical approach to this problem.

11 F. J. Arlinghaus, Phys. Rev. 157, 491 (1967).

12 K. H. Johnson and H. Amar, Phys. Rev. 139, A760 (1965). ${ }^{13} \mathrm{H}$. Amar, K. H. Johnson, and K. P. Wang, Phys. Rev. 148, 672 (1966).
} 
states per primitive cell of the crystal in partially filled bands. If we define $n \equiv n_{h}-n_{e}$, then

$$
-n=n_{e}-n_{h}=\left(\Omega / 4 \pi^{3}\right)\left(V_{e}-V_{h}\right) .
$$

A metal is said to be uncompensated if $n \neq 0$ and compensated if $n=0$.

Consider a perfectly ordered metallic compound having $s_{1}$ and $s_{2}$ atoms of atomic number $Z_{1}$ and $Z_{2}$, respectively, per primitive cell. Let there be $F$ full zones and $J$ hole zones ${ }^{14}$; since the total number of electrons must equal the total number of occupied states, we have ${ }^{15}$

$$
\left\{s_{1} Z_{1}+s_{2} Z_{2}\right\}=2 F+n_{e}+\left(2 J-n_{h}\right) .
$$

Denoting the quantity in braces by $N_{T}$, the total number of electrons per primitive cell, Eq. (2) becomes

$$
-n=n_{e}-n_{h}=N_{T}-2(F+J) .
$$

Therefore if $N_{T}$ is odd, the metal cannot be compensated and $n$ must be an odd integer. If $N_{T}$ is even, either $n$ must be even integer or the metal can be compensated if $N_{T}=2(F+J)$. However, for all nonmagnetic elements $^{14}$ and the one metallic compound ${ }^{8}$ which have been investigated to date, if $N_{T}$ is even, the metal is, in fact, compensated. For ordered $\beta$-brass, $Z_{\mathrm{Cu}}=29$, $Z_{\mathrm{Zn}_{\mathrm{n}}}=30, s_{\mathrm{Cu}}=s_{\mathrm{Zn}}=1$, so that it should be uncompensated and $n$ should be an odd integer. As we shall show later, ordered $\beta$-brass is uncompensated but we have not actually measured $n$, which can be obtained from the Hall effect in the high-field limit.

\section{B. High-Field Magnetoresistance Theory}

The theory of Lifshitz et al. ${ }^{16}$ and Lifshitz and Peschanskii ${ }^{17}$ has shown that in the high-field limit, the resistance of a metal single crystal is determined by its state of compensation and by topological properties of its Fermi surface. Table I summarizes the possible types of transverse magnetoresistance behavior; the longitudinal magnetoresistance (current density $\mathbf{J}$ parallel to field B) always saturates in the high-field limit. The table illustrates the major qualitative difference between compensated and uncompensated metals. In an uncompensated metal, the magnetoresistance saturates for a general field direction and quadratic behavior indicates the presence of open orbits perpendicular to the field but not perpendicular to the current. For a compensated metal, however, quadratic behavior is the general rule with saturation occur-

${ }^{14}$ See E. Fawcett, Advan. Phys. 13, 139 (1964) for definitions of electron and hole zones.

${ }^{15}$ This equation is a simple generalization of one given by Fawcett, Ref. 14.

${ }_{16}$ I. M. Lifshitz, M. I. Azbel, and M. I. Kaganov, Zh. Eksperim. i. Teor. Fiz. 31, 63 (1956) [English transl.: Soviet Phys.-JETP 4, 41 (1957)].

${ }_{17}$ I. M. Liftshitz and V. G. Peschanskii, Zh. Eksperim. i Teor. Fiz. 35, 1251 (1958); 38, 188 (1960) [English transls.: Soviet Phys.-JETP 8, 875 (1959); 11, 137 (1960)].

\begin{tabular}{|c|c|c|}
\hline State of compensation & Nature of orbits & $\begin{array}{c}\text { Magnetoresistance } \\
\text { behavior }\end{array}$ \\
\hline Uncompensated & All closed & Satures \\
\hline Compensated & All closed & \\
\hline Either & $\begin{array}{l}\text { Open orbits in } \mathbf{k} \text { space } \\
\text { making angle } \alpha \text { with } \mathbf{J}\end{array}$ & $B^{2} \cos ^{2} \alpha$ \\
\hline Either & $\begin{array}{l}\text { Nonintersecting open } \\
\text { orbits in } 2 \text { different } \\
\text { directions }\end{array}$ & Saturates \\
\hline
\end{tabular}

TABLE I. Transverse magnetoresistance in high-field limit.

ring essentially only when the open orbits are perpendicular to the current.

The result of a magnetoresistance investigation is a stereogram showing directions of field for which the magnetoresistance saturates and for which it is quadratic in field. There exist two different kinds of orbits, which have been called type A and type B. ${ }^{18}$ Periodic open orbits (type A) can exist in a one-dimensional region on a stereogram which is often a high-symmetry plane. Aperiodic open orbits (type B) can exist over a two-dimensional region on a stereogram near a highsymmetry axis; the direction of these open orbits is normal to the field and to the axis of high symmetry.

The $\cos ^{2} \alpha$ term in Table I results from a similarity transformation of the resistivity tensor from a coordinate system in which the open orbits lie along the $x$ axis to the sample coordinate system in which $\mathbf{J}$ is parallel to the $x^{\prime}$ axis. The angle between the $x$ and $x^{\prime}$ axes is $\alpha$. Alternatively, one can obtain this factor in another way as was shown by Klauder and Kunzler. ${ }^{19}$ If $\hat{\imath}_{0}$ is the direction of the open orbits and $\hat{\jmath}$ the direction of $\mathbf{J}$, the electric field due to these open orbits is

$$
\mathbf{E}_{0}=A \hat{\imath}_{0}\left(\hat{\imath}_{0} \cdot \hat{\jmath}\right) B^{2} \text {, }
$$

where $A$ is independent of the magnitude of $\mathbf{B}$ and varies slowly with the direction of $\mathbf{B}$. $\mathbf{B}$ need not be perpendicular to $\hat{\jmath}$ in the above equation. Assuming that the open orbits dominate the conductivity, the electric field, measured along $\hat{\jmath}$, is clearly proportional to $\left(\hat{\imath}_{0} \cdot \hat{\jmath}\right)^{2} B^{2}=B^{2} \cos ^{2} \alpha$. This means there should be a peak in the magnetoresistance as $\mathbf{B}$ passes through a one-dimensional open-orbit region. The height of the peak will diminish to zero as the angle between $\mathbf{J}$ and $\mathbf{B}$ approaches zero and the width of the peak will depend on the mobility of the open-orbit electrons.

\section{Energy-Band Calculations}

Qualitatively speaking the energy-band calculations resulting from the APW and Green's-function methods are quite similar. There are differences in detail, particularly in the position of the $d$ bands, but the general features of the Fermi surface predicted by the

${ }^{18}$ R. G. Chambers, The Fermi Surface (John Wiley \& Sons, Inc., New York, 1960), p. 100.

${ }_{19}$ J. R. Klauder and J. E. Kunzler, Phys. Rev. Letters 6, 179 (1961). 


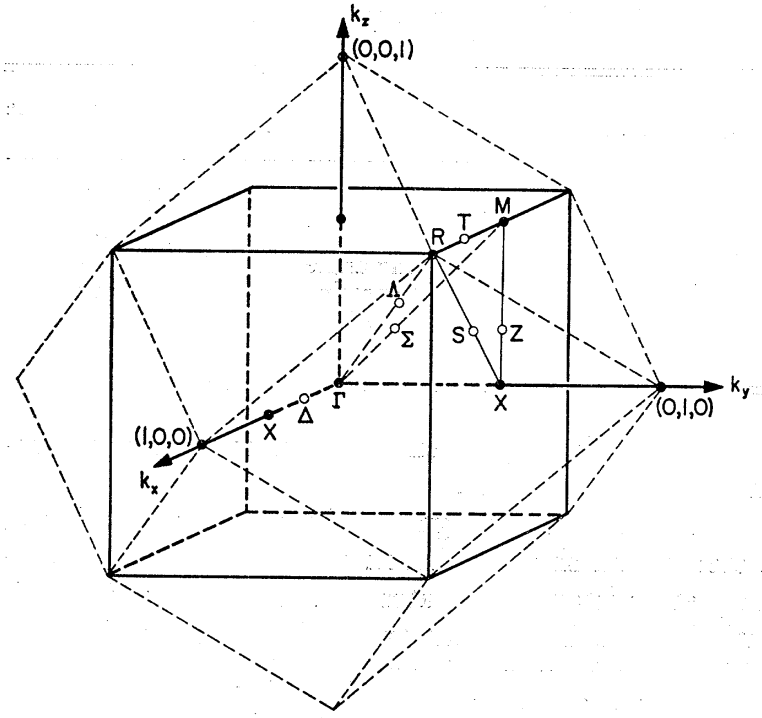

FIG. 1. First Brillouin zone for the simple cubic lattice showing symmetry points. The second zone is shown in dotted lines.

two calculations are identical. Figure 1 shows the first and second Brillouin zones for the cubic structure. The Fermi surface predicted by the calculations consists of hole pockets in the first zone centered on $R$ and a multiply connected electron surface in the second zone having necks, centered on $M$, in the $\{110\}$ Brillouin-zone planes. A sketch of the Fermi surface based on Arlinghaus's cross sections is given in the periodically extended zone scheme in Fig. 2. For later reference, the second-zone surface can be thought of topologically as a multiply connected set of "lenses" which are centered on the points $X$ and which intersect near the points $M$. The Fermi surface is not radically different from that based on a free-electron sphere which makes contact with the $\{110\}$ second-zone faces, but which does not

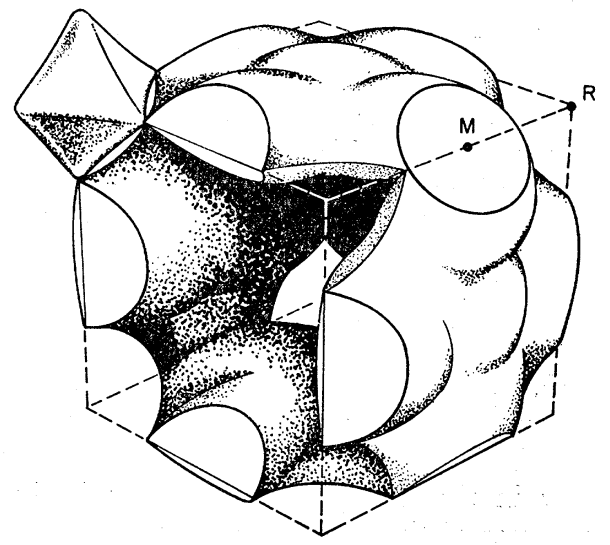

FIG. 2. Schematic drawing of Fermi surface of ordered $\beta$-brass in repeated zone scheme (based on cross sections of Arlinghaus, Ref. 11). The pocket centered on $R$ is the first-zone hole surface, and the multiply connected surface consists of electrons in the second zone. fill the corners of the zone near $R$. In particular, we wish to note that in the absence of spin-orbit coupling, the first and second bands are degenerate along the line $T$. This causes the first and second bands to stick together as is shown schematically in Fig. 3.

\section{EXPERIMENTAL PROCEDURE AND RESULTS}

\section{A. Samples and Experimental Procedure}

Samples of $\beta^{\prime}$-CuZn were prepared by melting together high-purity copper and zinc in an evacuated quartz tube and quenching to room temperature. A long annealing followed just below the solidus line. The ingot was then slowly cooled to a temperature slightly above the $\beta+\gamma$ region and quenched rapidly to room temperature. This produced very large grains from which cylindrical samples of approximate dimensions $2 \mathrm{~mm} \times 10 \mathrm{~mm}$ were spark cut. Single crystals of alloys prepared in this way were examined by $\mathrm{x}$-ray rotation photographs taken in liquid helium at $4.2^{\circ} \mathrm{K}$ to ensure that no martensitic transformation occurs. ${ }^{4}$ The orientation and resistivity ratios, $\rho\left(295^{\circ} \mathrm{K}\right) / \rho\left(4.2^{\circ} \mathrm{K}\right)$, of the

TABLE II. Orientations and resistance ratios of the various samples.

\begin{tabular}{cc}
\hline \hline Current axis & Resistance ratio \\
\hline$\langle 100\rangle$ & 367 \\
$\langle 110\rangle$ & 380 \\
$\langle 210\rangle$ & 418 \\
$\langle 310\rangle$ & 385 \\
$\langle 610\rangle$ & 410 \\
\hline
\end{tabular}

samples are given in Table II. The current axes are along the directions shown to within $1 \mathrm{deg}$. Current and voltage leads were soldered to the samples with low-melting solders, care being taken not to heat the samples into the $\beta+\gamma$ region of the phase diagram.

The magnetoresistance was measured in the field of a 150-kG water-cooled solenoid at the National Magnet Laboratory. ${ }^{20}$ The sample holder, which is described in detail elsewhere, ${ }^{21}$ had two degrees of freedom so that the field could be pointed in any crystallographic direction for an arbitrary-current axis. This enables one to investigate the field dependence of the magnetoresistance with a small number of samples, which is not the case if only transverse magnetoresistance is measured.

Figure 4(a) illustrates the two degrees of freedom of the sample holder in the laboratory coordinate system Oxyz. The magnetic field is directed along $O z$. The sample is tipped at an angle $\phi$ from the $x$ axis, about which it can rotate, $\psi$ being the angle of rotation measured from the $O x z$ plane. The tip angle $\phi$, which can be varied from $-90^{\circ}$ to $+90^{\circ}$, can be adjusted only at $\psi=0$. Figure $4(\mathrm{~b})$ is a stereogram in the sample co-

${ }^{20}$ Supported by the U. S. Air Force Office of Scientific Research. ${ }^{21}$ D. J. Sellmyer, Rev. Sci. Instr. 38, 434 (1967). 


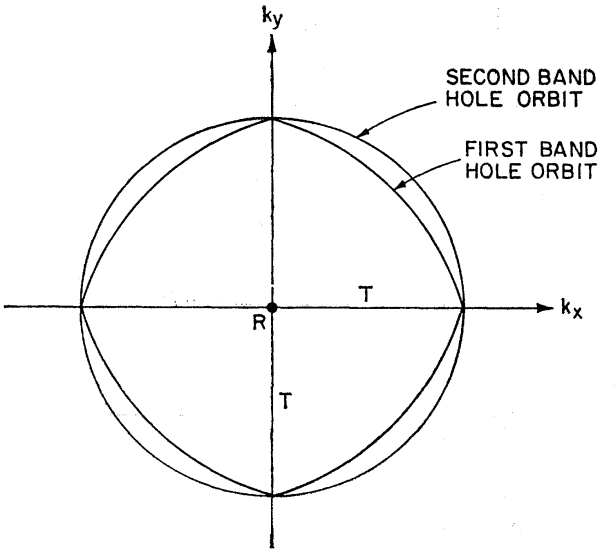

FIg. 3. Fermi surface cross sections in cubic-zone face showing degeneracy along line $T$. (Based on cross sections of Arlinghaus, Ref. 11.)

ordinate system $0 \xi \eta \zeta .0 \xi$ is the axis of the sample [Fig. 4(a)], $\mathbf{B}(0)$ is the magnetic field at $\psi=0$. By varying $\psi$ and $\phi$, the whole stereogram can in principle
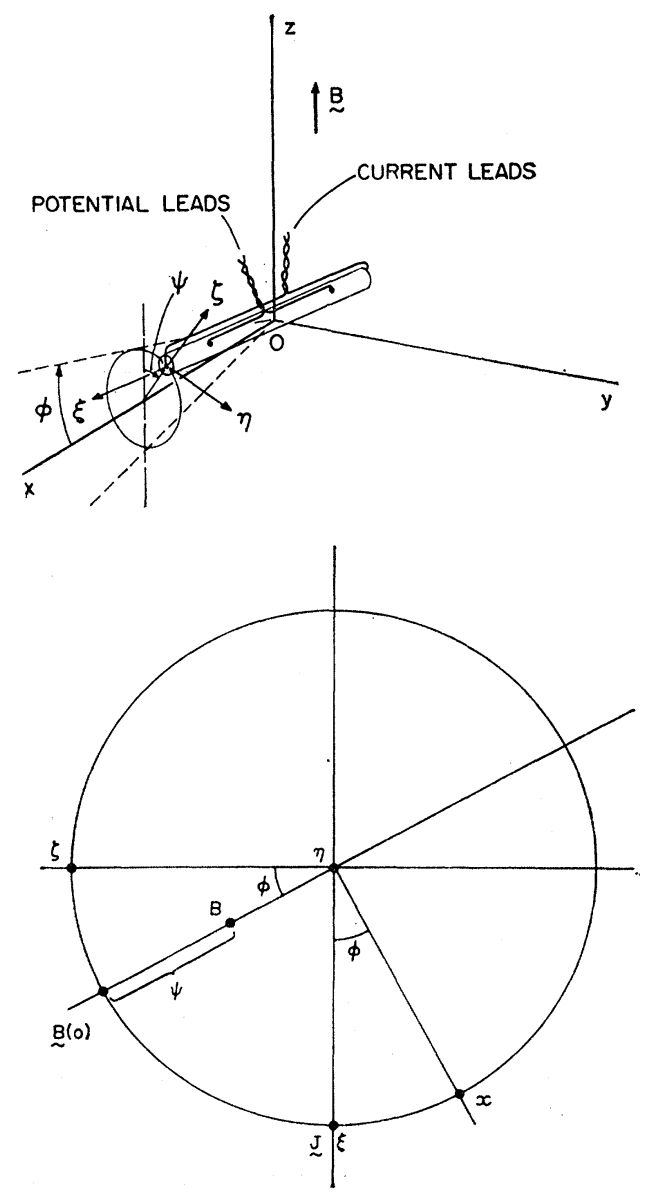

Frg. 4. (a) Sample rotation and tipping geometry. The laboratory coordinate system in $O x y z$ and the sample coordinate system is $0 \xi \eta \zeta$. (b) Stereogram showing the effect in the sample coordinate system of rotating and tipping the sample in the magnetic field.

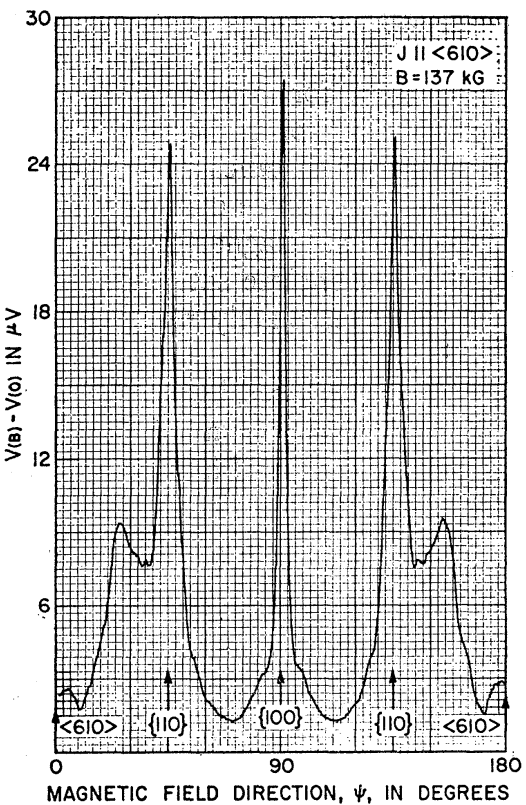

FIG. 5. Photograph of magnetoresistive-voltage rotation plot for $\phi=90^{\circ}$. The sharpness of the peaks gives an indication of the effective values of $\omega_{c} \tau$.

be covered with one sample. However, as the angle between $\mathbf{J}$ and $\mathbf{B}$ approaches zero, open orbits can be missed, as is shown by Eq. (4) and the discussion below it.

The magnetoresistive voltages $V(B)$ were measured with a Keithley Model 148 nanovoltmeter. Rotation plots were recorded continuously on an $X-Y$ recorder as the angle $\psi$ was rotated by a motor at the top of the cryostat; the $X$-axis signal was derived from a linear 10-turn potentiometer which was coupled to the motor. The sample current was typically between 1 and $2 \mathrm{~A}$ and was held constant to better than 1 part in $10^{4}$, the supply being a Princeton Applied Research Model TC602 CR. All measurements reported here were made at $4.2^{\circ} \mathrm{K}$ since it was determined experimentally that lowering the temperature to $1.2^{\circ} \mathrm{K}$ increased the magnetoresistance by a negligible amount.

\section{B. Magnetoresistance Results}

It is first necessary to demonstrate that the magnetoresistance is actually being measured in the high-field region. A rough estimate of $\left\langle\omega_{c} \tau\right\rangle_{\text {av }}$ can be made on the basis of free-electron theory. If we assume three conduction electrons per unit cell and consider a crystal of resistance ratio $410,\left\langle\omega_{c} \tau\right\rangle_{\mathrm{av}}=14$ at $150 \mathrm{kG}$. Figure 5 shows a photograph of a typical rotation plot, in this case a longitudinal-to-transverse run on the $\langle 610\rangle$ sample. Using the theory of Lifshitz et al. ${ }^{17}$ the width of the maximum as the field traverses a one-dimensional region of open orbits is inversely proportional to $\left\langle\omega_{c} \tau\right\rangle_{\text {av }}$. The sharpness of the peaks in Fig. 5 when B is 
in $\{110\}$ or $\{100\}$ planes suggests that at $137 \mathrm{kG}$, $\left\langle\omega_{c} \tau\right\rangle_{\mathrm{av}}$ is indeed larger than unity. Furthermore, the theory predicts that for any state of compensation and any configuration of open or closed orbits, the longitudinal magnetoresistance saturates in the highfield region. Figure 7, curve d, shows the field dependence of the longitudinal magnetoresistance for $\psi=0^{\circ}$ on Fig. 5. The nearly complete saturation at $150 \mathrm{kG}$ indicates that we are reasonably well into the high-field region.

The transverse-magnetoresistance rotation plots of several of the samples are shown in Fig. 6. The magnetoresistance is low for a general field direction and peaks occur only when the field lies in a symmetry plane or is close to a high symmetry axis. From this we conclude

(o)
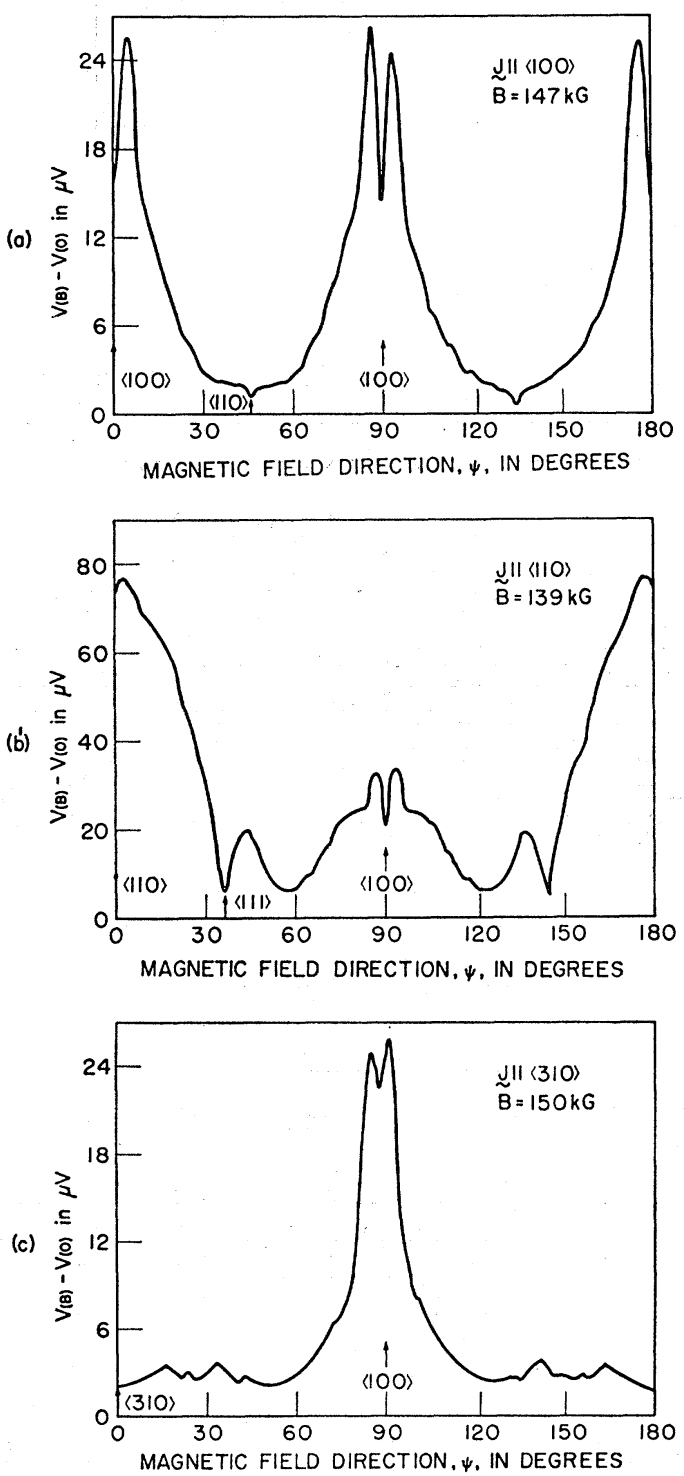

FIg. 6. Transverse magnetoresistive voltages for the $\langle 100\rangle$, $\langle 110\rangle$, and $\langle 310\rangle$ samples. that ordered $\beta$-brass is an uncompensated metal, in agreement with the discussion of Sec. IIA.

The field dependences of the magnetoresistance at several points of the rotation plots of Figs. 5 and 6 are shown in Figs. 7 and 8. Let the field dependence of the magnetoresistance be given by

$$
\frac{\Delta \rho}{\rho}=\frac{\rho(B)-\rho(O)}{\rho(O)}=a B^{m},
$$

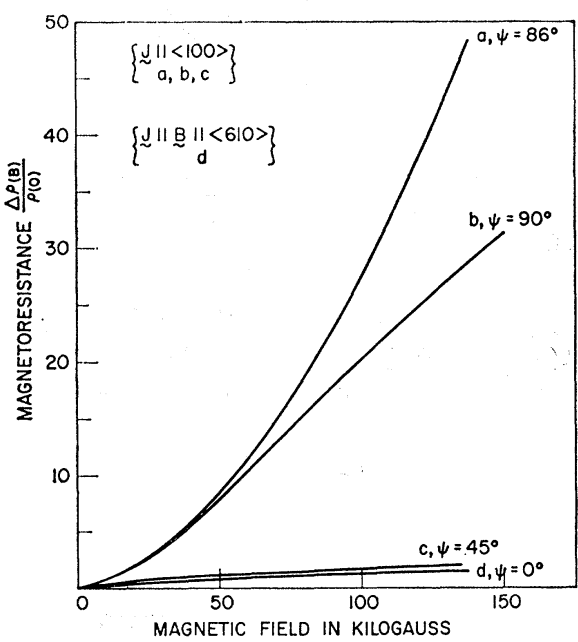

FIG. 7. Magnetoresistance as a function of magnetic field for several field directions on the rotation plots of Figs. 5 and 6(a). The angles $\psi$ at which the field dependence was measured are shown at the right of the curves.

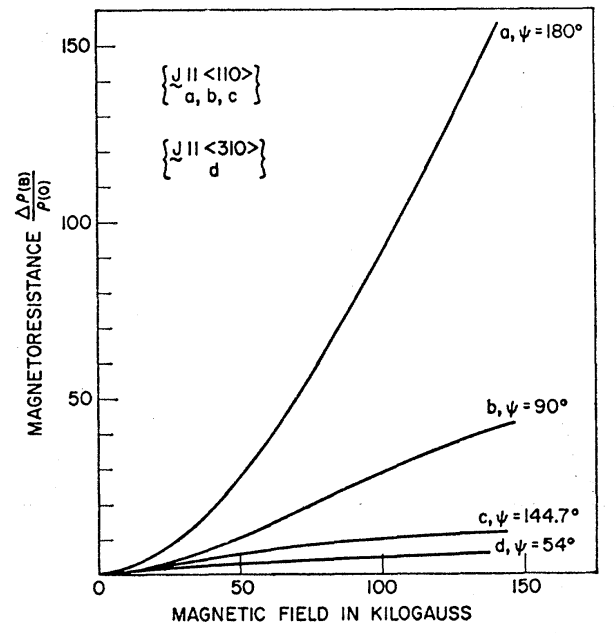

FIG. 8. Magnetoresistance as a function of magnetic field for several field directions on the rotation plots of Figs. 6(b) and 6(c). The angles $\psi$ at which the field dependence was measured are shown at the right of the curves.

where $a$ is a constant. Table III lists $m$ at the highest value of the field for the curves of Figs. 7 and 8 . In what follows we will call the field dependence "quadratic" if $m \gtrsim 1.8$ and "saturating" if $m \lesssim 0.5$; it seems reason- 
able to assume that with samples having less deviation from perfect stoichiometry and with less mosaic structure, the theoretically predicted values of $m=2$ or 0 would obtain where we have found $m \simeq 1.8$ or 0.5 , respectively.

There are two puzzling features of the results we have presented thus far. The first one is the lack of saturation or quadratic dependence when $\mathbf{B} \|\langle 100\rangle$, as illustrated by curves $\mathrm{b}$ of Figs. 7 and 8, corresponding to Figs. 6(a) and 6(b), respectively. The following test was made to decide whether this behavior was genuine or due to a small misalignment of the field. It was possible to make the peaks on either side of $\psi=90^{\circ}$, Fig. 6(a), have the same height, by decreasing the tip angle $\phi$ by $1.6^{\circ}$. The effect of decreasing $\phi$ further by $1.6^{\circ}$ was simply to reflect the central portion of the figure about the $\psi=90^{\circ}$ point. No enhancement of the saturation was effected by the above procedure. Therefore, unless there is a dip having a width of only tenths of a degree, we conclude that the true magnetoresistance behavior when $\mathbf{B} \|\langle 100\rangle$ is approximately

TABLE III. Field dependence of the magnetoresistance.

\begin{tabular}{cccc}
\hline $\begin{array}{c}\text { Figure and } \\
\text { curve }\end{array}$ & $\begin{array}{c}\text { Current direction } \\
\mathbf{J}\end{array}$ & $\begin{array}{c}\text { Plane or } \\
\text { direction of } \mathbf{B}\end{array}$ & $m$ \\
\hline $7 \mathrm{a}$ & $\langle 100\rangle$ & $4^{\circ}$ from & 1.83 \\
& & $\langle 100\rangle$ in $\{100\}$ & \\
$7 \mathrm{~b}$ & $\langle 100\rangle$ & $\langle 100\rangle$ & 0.93 \\
$7 \mathrm{c}$ & $\langle 100\rangle$ & $\langle 110\rangle$ & 0.43 \\
$7 \mathrm{~d}$ & $\langle 610\rangle$ & $\langle 610\rangle$ & 0.31 \\
$8 \mathrm{a}$ & $\langle 110\rangle$ & $\langle 110\rangle$ & 1.84 \\
$8 \mathrm{~b}$ & $\langle 110\rangle$ & $\langle 100\rangle$ & 0.98 \\
$8 \mathrm{c}$ & $\langle 110\rangle$ & $\langle 111\rangle$ & 0.30 \\
$8 \mathrm{~d}$ & $\langle 310\rangle$ & $\{310\}$ & 0.54 \\
\hline
\end{tabular}

linear. The second feature concerns the small dip seen at $\mathbf{B} \|\langle 110\rangle$ in Fig. 6(b). This is due to a small misalignment of the crystal relative to the field, as we shall see in the course of the following discussion.

Figure 9 is a stereographic projection of the results obtained with a $\langle 210\rangle$ sample, and Fig. 10 reproduces these results for four different tip angles. We wish to make several points with regard to these figures:

(1) The asymmetry of Figs. 10(b), 10(c), and 10(d) near $\psi=90^{\circ}$ and the lack of a dip at $\psi=90^{\circ}$ in Fig. 10(a) indicate that there is a slight misalignment of the tipping plane away from (001); we estimate this to be $\sim 2^{\circ}$. This means that $\mathbf{B}$ misses being parallel to [001] at $\psi=90^{\circ}$ by $\sim 2^{\circ}$.

(2) The highest and sharpest peaks occur when B lies in $\{110\}$ or $\{100\}$ planes.

(3) The magnetoresistance exhibits "volcanic" behavior in Figs. $10(\mathrm{c})$ and $10(\mathrm{~d})$ at $\psi \sim 35^{\circ}$ as B nears the [111] direction. Figure 9 shows that as $\phi$ decreases from positive to negative values the peaks adjacent to the dip near [111] leave, for example, the (011) plane. The area near the $[1 \overline{1} 1]$ direction has been carefully

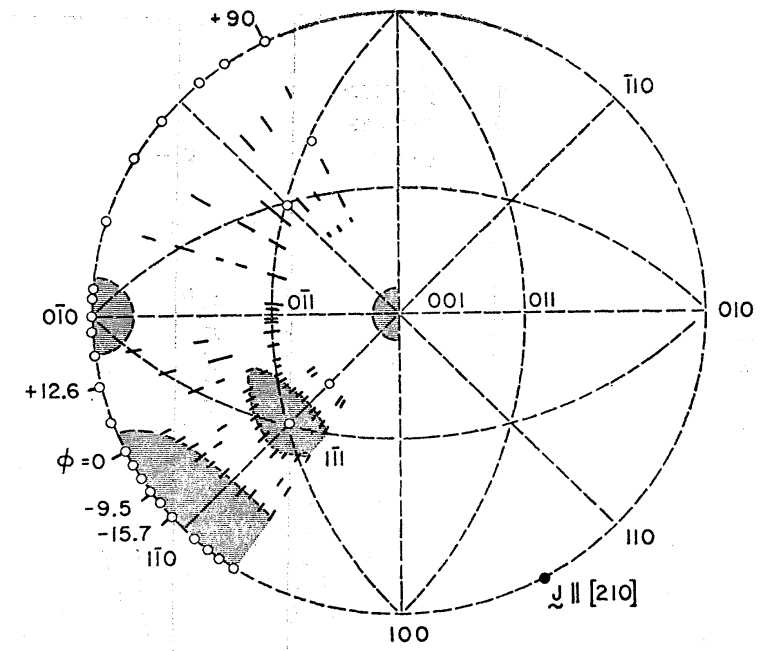

Fig. 9. Stereographic projection showing positions of magnetoresistance peaks (-) and dips (0) for rotation runs on $\langle 210\rangle$ crystal. No peaks are shown in the vicinity of [001]; when the field is rotated in a $\{110\}$ or $\{100\}$ plane, only the positions of the dips are shown. Tip angles for the plots of Fig. 10 are shown at the periphery of the stereogram, where $\psi=0^{\circ}$. The length of the lines denoting peaks attempts to convey qualitatively the width of the peaks.

investigated and in the stereogram we have crosshatched the area enclosed by the peaks near [111] since it appears to be a two-dimensional region of open orbits.

(4) Figures 10(c) and 10(d) show that the dip at $\psi=0^{\circ}$ becomes less pronounced as the field-rotation plane approaches the (110) plane; in fact, when the field is rotated in the (110) plane the resulting plot is nearly identical to the transverse rotation plot of Fig. 6(b) except that the small dip at $\psi=0^{\circ}$ in Fig. 6(b) is absent. This proves our earlier assertion that the small dip in Fig. 6(b) at $\psi=0$ is simply the result of a small misalignment of the crystal.

(5) When the field is rotated in the (100) plane, the resulting plot is similar to the transverse-rotation plot of Fig. 6(a) except that in this case there is a spike when $\mathbf{B}$ is near [0 $\overline{1} 1]$. The absence of a peak at this position in Fig. 6(a) is understandable in terms of the $\cos ^{2} \alpha$ factor of Table I. These last two points [i.e., (4) and (5)] provide a demonstration of the efficacy of our type of sample holder in reducing the number of sample orientations needed, at least for uncompensated metals.

(6) Figures 9 and 10 show that when $\mathbf{B}$ is in the vicinity of $\langle 100\rangle$ and $\langle 110\rangle$ directions the magnetoresistance is quite high. This has been indicated in Fig. 9 by the shaded areas. The size of the $\langle 100\rangle$ area was taken from the width of the two peaks near $\langle 100\rangle$ in Figs. 6(a) and 6(b) and from similar peaks in the relevant rotation plots of the $\langle 210\rangle$ crystal. For the [110] area the peak positions near [110] were simply connected together to form a two-dimensional region; for $\phi=+6.3^{\circ}$ the peak near $\psi=0^{\circ}$ had vanished, 


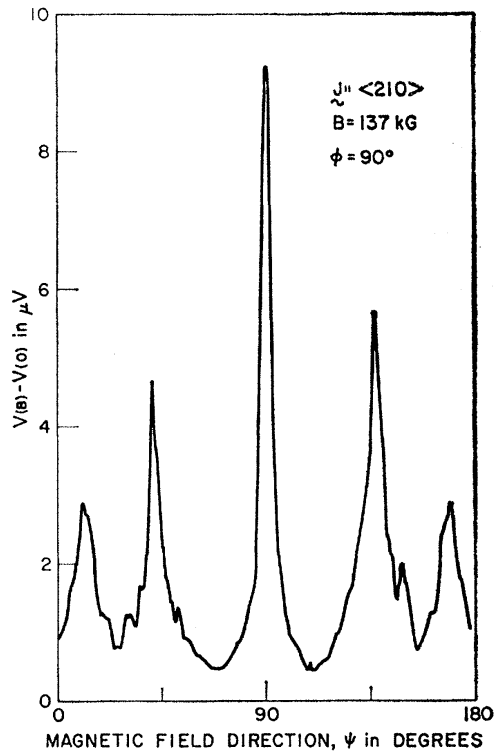

(a)

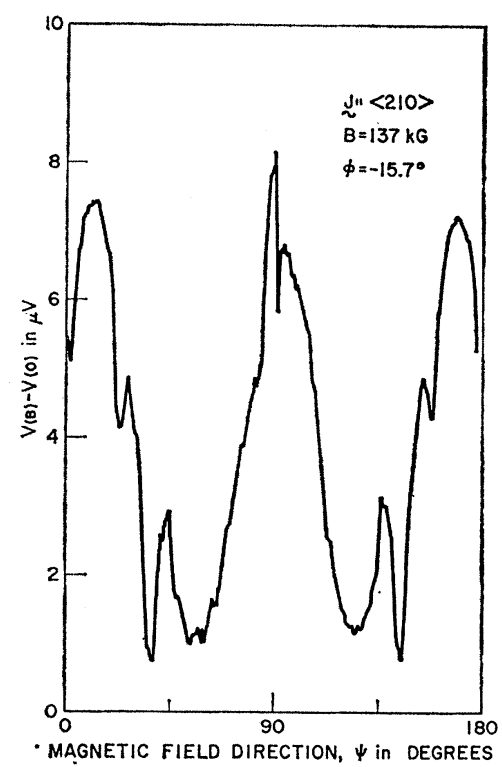

(d)

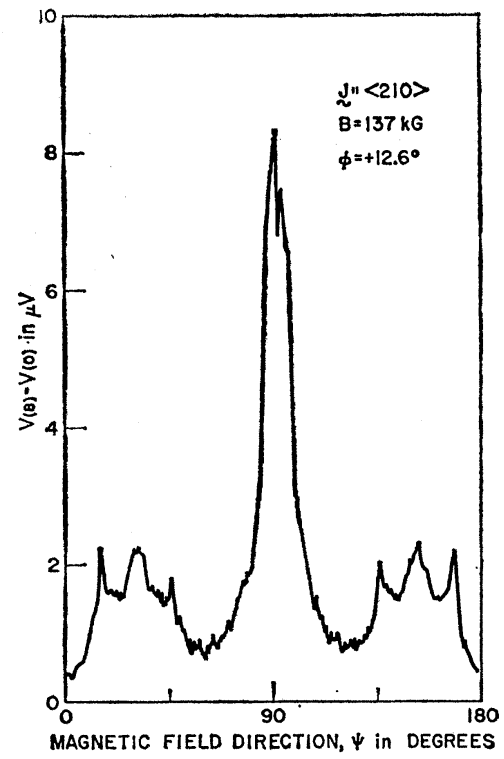

(b)

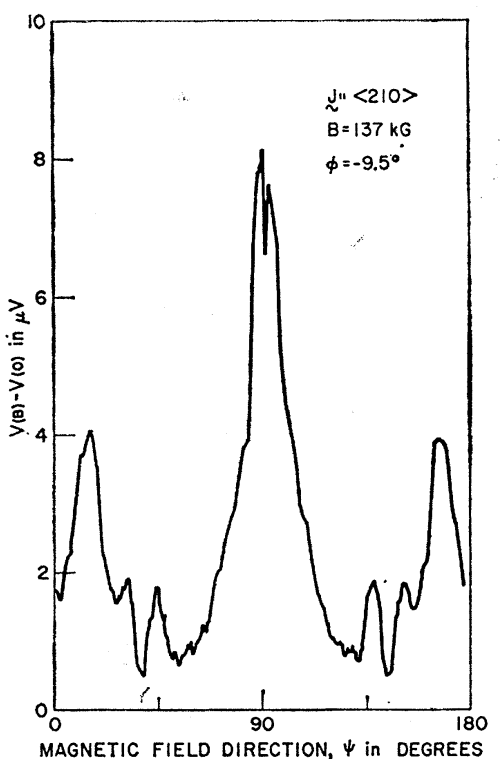

(c)

FIG. 10. Magnetoresistance-voltage plots for nonzero tip angles for $\langle 210\rangle$ crystal. The tip angles $\phi$ of these plots are shown in Fig. 9.

(7) Although the occurrence of narrow peaks is strongest in the $\{110\}$ and $\{100\}$ planes, there are also smaller peaks in other planes as shown in Fig. 9. These smaller peaks occur in higher-order planes such as $\{210\}$ and $\{111\}$ planes.

(8) Several of the other samples were investigated for various tip angles with results in agreement with those of Figs. 9 and 10.

\section{DISCUSSION}

Before attempting to compare the results with the Fermi surface predicted by the energy-band calculations we must consider the effect of the degeneracy of the first and second bands along the line $T$. In metals with small energy gaps it is possible, in the presence of high magnetic fields, for electrons to tunnel from one sheet of Fermi surface to another. Blount ${ }^{22}$ has shown that the probability for such magnetic breakdown is

$$
P=\exp \left(-B_{0} / B\right)
$$

where, in the case of nearly free electrons, the chararacteristic breakdown field is given by

$$
B_{0}=\pi(\Delta \epsilon)^{2} c / 4 \hbar e v_{x} v_{y} \text {. }
$$

The field is taken along the $z$ direction, $v_{x}$ and $v_{y}$ are

${ }^{22}$ E. I. Blount, Phys. Rev. 126, 1636 (1962). 
components of velocity parallel and perpendicular to the zone boundary, and $\Delta \epsilon$ is the energy gap between the two bands. In the case where $v_{x}=v_{y}$ and there is no component of $\mathbf{v}$ parallel to $\mathbf{B}$, the above equation reduces to

$$
B_{0}=\pi(\Delta \epsilon)^{2} m c / 4 \hbar \epsilon \epsilon_{F},
$$

where $\epsilon_{F}$ is the Fermi energy and $m$ is the electronic mass. Let us assume for the moment that the spinorbit interaction does not lift the degeneracy on the line $T$. Using Eq. (8), we wish to estimate roughly the angular region, measured from the corner of zone $R$, over which magnetic breakdown might be expected. To do this we make the $a d$ hoc assumption that the energy gap decreases linearly with angle from the line $S$ to the line $T$. The energy gap on the line $S$ at the Fermi surface is, according to Arlinghaus, approximately 0.7 $\mathrm{eV}$. Let $\mathbf{B}$ be tipped away from a $\langle 100\rangle$ direction in a $\{100\}$ plane and let $\theta$ be the angle between $\mathbf{B}$ and the $\langle 100\rangle$ direction. Taking $\epsilon_{F}=7.45 \mathrm{eV}^{23}$ and $B=150 \mathrm{kG}$, we obtain for the angle at which $B=B_{0}, \theta_{0} \simeq 8^{\circ}$ corresponding to $\Delta \epsilon\left(\theta_{0}\right) \simeq 0.13 \mathrm{eV}$. Therefore, on the basis of these admittedly crude arguments, we would expect at $150 \mathrm{kG}$ a small angular region of magnetic breakdown to exist near the lines $T$ unless the spin-orbit splitting is of the order of tenths of an electron volt.

The symmetry of the degenerate band which intersects the Fermi level along the line $T$ is $T_{5},{ }^{11}$ and group theory predicts a nonvanishing spin-orbit splitting for a state of this symmetry. Let $b$ be the length of the cubic-Brillouin-zone edge; the APW calculation of Arlinghaus shows that at the point $\mathbf{k}=\frac{1}{2}\left(1,1, \frac{1}{2}\right) b$, which is close to the Fermi surface, the wave function of symmetry $T_{5}$ is a complicated admixture of plane wave plus atomic $p$ and $d$ states. It is, therefore, impossible to estimate from relativistic atomic calcula-

TABLE IV. Open-orbit characteristics.

\begin{tabular}{|c|c|c|c|c|}
\hline Type & Direction & $\begin{array}{c}\text { Plane } \\
\text { of B }\end{array}$ & $\begin{array}{c}\text { Orbits vanish; } \\
\mathbf{B}_{c}, \theta_{c}{ }^{\mathbf{a}}\end{array}$ & Characteristics $^{b}$ \\
\hline $\mathrm{I}$ & $\langle 100\rangle$ & $\{100\}$ & $\langle 100\rangle$ & $N H N L$ \\
\hline III & $\begin{array}{l}\langle 100\rangle \\
\langle 110\rangle\end{array}$ & $\{100\}$ & $\begin{array}{c}\langle 100\rangle,\langle 110\rangle \\
\langle 110\rangle\end{array}$ & $\begin{array}{l}\text { NLNLHL } \\
L N H N\end{array}$ \\
\hline IV & $\langle 110\rangle$ & $\{110\}$ & $\begin{array}{r}\langle 110\rangle \text { and } \\
\theta>30.6^{\circ}\end{array}$ & HHLNLNL \\
\hline V & $\langle 110\rangle$ & $\{110\}$ & $\begin{array}{l}\text { from }\langle 110\rangle \\
\langle 110\rangle \text { and } \\
\theta>31.9 \text { from } \\
\langle 100\rangle\end{array}$ & $H L N L H N$ \\
\hline VI & $\langle 210\rangle$ & $\{210\}$ & $\begin{array}{c}\langle 100\rangle \text { and } \\
\theta>26.9 \text { from } \\
\langle 100\rangle\end{array}$ & $\begin{array}{l}H L N L N L H L N \\
\quad L H N\end{array}$ \\
\hline VII & $\langle 310\rangle$ & $\{310\}$ & $\begin{array}{l}\langle 100\rangle \text { and } \\
\theta>25.9 \text { from } \\
\langle 100\rangle\end{array}$ & $\begin{array}{l}\text { (generalization } \\
\text { of type VI) }\end{array}$ \\
\hline
\end{tabular}

\footnotetext{
${ }_{\mathrm{A}} \mathrm{B}_{\mathrm{c}}$ and $\theta_{c}$ denote directions and critical angles at which the open orbits are lost.

b $L$ indicates orbit traverses "lens" in second band; $H$ indicates orbit crosses $\{100\}$ second-band hole orbit around $R ; N$ indicates orbit crosses (110) second-band neck orbit around $M$.

${ }^{23}$ J.-P. Jan, Can. J. Phys. 44, 1787 (1966).
}

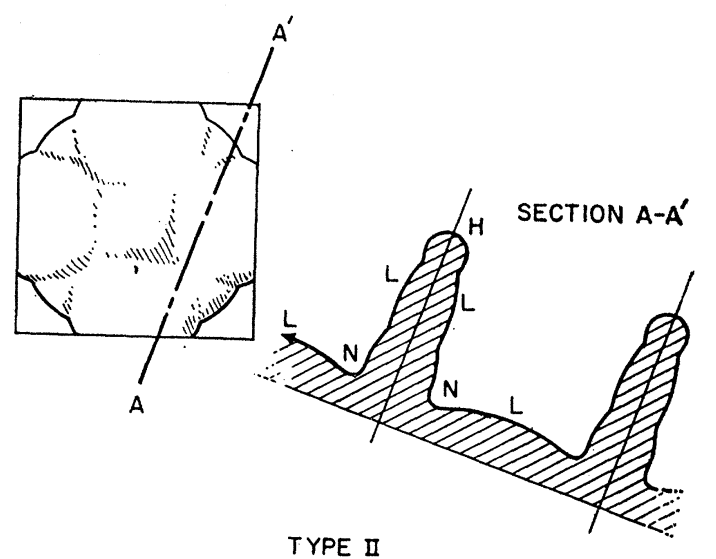

TYPE II

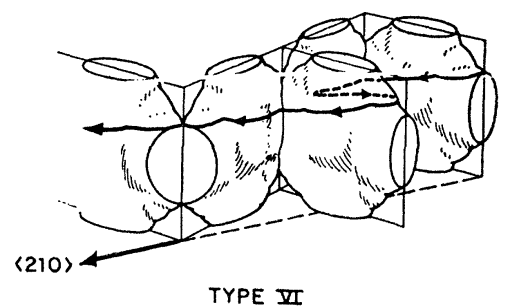

Frg. 11. Diagrams showing the topology of two of the open orbits listed in Table IV (not to scale). (a) Type-II orbits in a $\langle 100\rangle$ direction. The letters on various parts of the orbit are explained in Table IV. (b) Type-VI open orbit in a $\langle 210\rangle$ direction.

tions, ${ }^{24}$ for example, the magnitude of the spin-orbit splitting in the solid; the coefficients and the radial parts of the APW wave functions are needed to compute the splitting. ${ }^{25}$ Under these circumstances we shall simply compare our results with the simple model of Fig. 2, bearing in mind that whenever an orbit passes near the line $T$ an interband transition may occur.

From a topological viewpoint, the Fermi-surface model of Fig. 2 is somewhat complicated and it is difficult to visualize the intersections of planes, which are imagined to be normal to $\mathbf{B}$, with such a surface. With the help of a three-dimensional model, we have been able to discover some of the possible types of periodic open orbits. Table IV lists these types of orbits and gives the directions for which they exist and their topological characteristics. Figure 11 shows schematically two of the types of open orbits listed in Table IV. ${ }^{26}$ The critical angles $\theta_{c}$ at which certain orbits are lost were calculated by taking from Arlinghaus the "radius" of the $\{100\}$ second-band hole orbits to be $0.27 b$.

${ }^{24}$ F. Herman and S. Skillman, Atomic Structure Calculations (Prentice-Hall, Inc., Englewood Cliffs, New Jersey, 1963).

${ }^{25} \mathrm{P}$. Decicco (private communication).

${ }^{26} \mathrm{~J}$. C. Wood, J.-C. Chevrier, and R. V. Coleman, University of Virginia Technical Report (unpublished); These authors have discussed the thickness of the type-I and type-II open orbits as a function of field direction. 
The results of the previous section make it clear that the Fermi surface of ordered $\beta$-brass is multiply connected and that the $\{100\}$ and $\{110\}$ planes are onedimensional regions of open orbits. The observation of strong open-orbit effects whenever B lies in these planes is consistent with the behavior predicted by the Fermisurface model, as is shown in Table IV. This agreement, when coupled with the dHvA measurements of several of the smaller orbits predicted by the model, provides rather convincing evidence of the validity of the general features of the model.

Except for the peaks denoting the boundaries of the shaded regions, all of the additional peaks in Fig. 9 occur in either $\langle 111\rangle$ or $\langle 210\rangle$ zones. It is possible that these peaks are the result of higher-order open orbits which result from a compounding of $\langle 110\rangle$ primary open orbits. The $\langle 110\rangle$ orbits are taken as primary open orbits because they are the strongest ones observed experimentally and because a prominent feature of the Fermi-surface model is the necks in the $\langle 110\rangle$ directions, without which the second-band sheet would not be multiply connected. The idea of com-
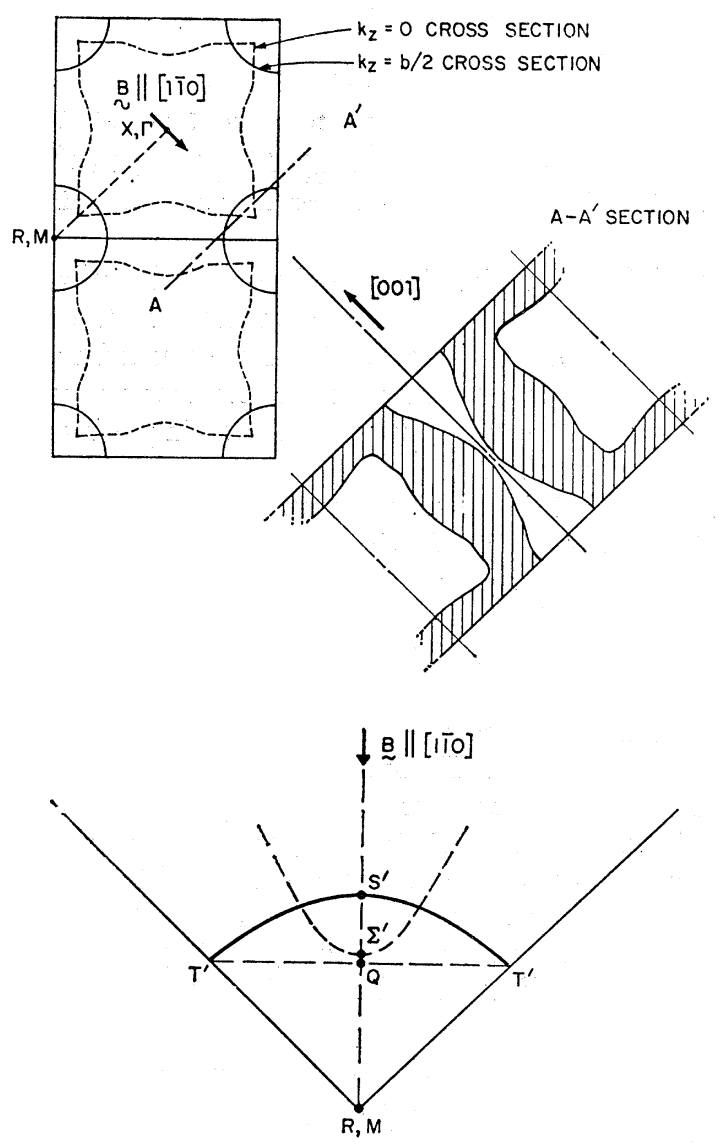

FIG. 12. (a) Diagram showing the topology of type-I open orbits in the [001] direction (not to scale). $k_{z}$ is measured from $\Gamma$ along [001]. (b) Diagram showing the criterion for the existence of type-I open orbits in the [001] direction (not to scale). If $M \Sigma^{\prime}$ $>R S^{\prime}>R Q$, the orbits would not exist. pounding primary open orbits to form higher-order open orbits was introduced by Klauder et al. ${ }^{27}$ who showed the existence of orbits up to order 6 by compounding the $\langle 111\rangle$ neck directions in copper. We have not attempted to study such higher-order open orbits in detail because they are rather weak in our samples; extremely large values of $\left\langle\omega_{c} \tau\right\rangle_{\mathrm{av}}$ are needed to study these orbits effectively.

We now consider the behavior of the magnetoresistance when $\mathbf{B}$ is along [110]. A convenient way to discuss this is to subscript the field-dependence exponent $m$ with the current direction $\hat{\jmath}$ so that, for example, $m_{110} \rightarrow 2$ implies that for $\hat{\jmath} \|[110], m \rightarrow 2$ as $B \rightarrow 150$ $k \mathrm{G}$. The results of the previous section give $m_{110} \rightarrow 2$ and $m_{001} \rightarrow 0$ when $\mathbf{B} \|[1 \overline{1} 0]$. Table $\mathrm{V}$ lists the various possible open-orbit combinations and the resulting theoretical behavior of $m_{110}$ and $m_{001}$ in the high-field limit. A consideration of Tables IV and V and the observed behavior when $\mathbf{B} \|[1 \overline{1} 0]$ (Table III) points out a distinct contradiction between the theoretical model and experiment. Table IV shows that type-I open orbits should exist along [001] when $\mathbf{B} \|[1 \overline{1} 0]$, and this indicates case (b) of Table V, whereas the experimental results are obviously in accordance with case (c) of Table V. This raises the question of whether (a) it is possible to eliminate the type-I orbit, perhaps by slightly deforming the Fermi-surface model, and (b) it is possible to obtain $\langle 110\rangle$ directed open orbits by taking into account magnetic breakdown.

Figure 12 illustrates the topology of the type-I open orbits when $\mathbf{B} \|[1 \overline{1} 0]$. It is clear from Fig. 12(b) that these orbits could not exist if $M \Sigma^{\prime}>R S^{\prime}>R Q$; the $k_{z}=0$ and $k_{z}=b / 2$ Fermi-surface cross sections would not overlap and no intersections of planes parallel to (110) with the Fermi surface could support type-I open orbits. Table VI lists the numerical values provided by various band-structure calculations. The values of $R T^{\prime}$, $M T^{\prime}$, and $M \Sigma^{\prime}$ given by the single-OPW (orthogonalized-plane-wave) model are quite unreliable; the freeelectron sphere is almost tangent to the $\{110\}$ Brillouin zone faces so that the smallest amount of lattice potential would change these values drastically. Values quoted from Arlinghaus ${ }^{11}$ or Johnson and Amar ${ }^{13}$ are read from their graphs. In this connection, it is not clear how the last authors drew the energy bands along the line $S$ since apparently they did not compute the energy

TABLE V. Magnetoresistance when $\mathbf{B} \|[1 \overline{1} 0]$.

\begin{tabular}{cccc}
\hline & Open-orbit directions & $m_{110}$ & $m_{001}$ \\
\hline (a) & None & 0 & 0 \\
(b) & {$[001]$} & $0^{\mathrm{a}}$ & 2 \\
(c) & {$[110]$} & 2 & $0^{\mathrm{a}}$ \\
(d) & Nonintersecting orbits $\|[110]$ and & 0 & 0 \\
& {$[001]$ or $\|$ to any 2 directions } & & \\
& in (110)
\end{tabular}

\footnotetext{
a Saturation caused by $\cos ^{2} \alpha$ factor.

${ }^{27}$ J. R. Klauder, W. A. Reed, G. F. Brennert, and J. E. Kunzler, Phys. Rev. 141, 592 (1966).
} 
except at the end points $R$ and $X .{ }^{12}$ The preliminary results of Taylor ${ }^{28}$ are obtained from a pseudopotential interpolation scheme, fitting extremal cross sections of the Fermi surface observed by Jan et al., ${ }^{4}$ using the de Haas-van Alphen effect. The last three rows of Table VI are in agreement with $R S^{\prime}>M \Sigma^{\prime}>R Q$, and Fig. 12(b) has been drawn to conform with these conditions. The calculations, therefore, predict that the type-I orbits do exist in fact, and imply that the thickness of the band of open orbits when $\mathbf{B} \|\langle 110\rangle$ is $S^{\prime} \Sigma^{\prime}$. If the calculations are correct we should have to seek another explanation for our failure to observe open orbits parallel to [001] when $\mathbf{B} \|[1 \overline{1} 0]$. From Fig. 12(b) it is seen that at least some of the open orbits have to intersect the $\operatorname{arc} S^{\prime} T^{\prime}$ fairly close to $T^{\prime}$. Depending on how the energy gap varies from $T^{\prime}$ to $S^{\prime}$, and upon the exact shape of the "arc" $T^{\prime} S^{\prime}$, it is possible that the type-I orbits are effectively cut off by magnetic breakdown. We conclude that the type-I open orbits do not exist either because the true Fermi-surface dimensions do not permit it or because they are eliminated by magnetic breakdown.

We now take up the question of the existence of open orbits parallel to [110]. Such orbits are easily envisioned

TABLE VI. Distances in the second band [Fig. 12(b)]. The unit is the length of the cubic zone edge.

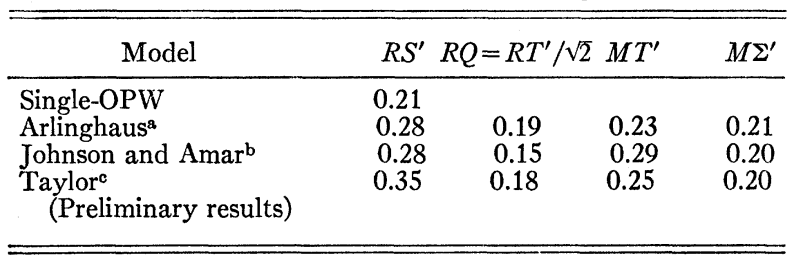

a Reference 11. b Reference 13. o Reference 28.

if magnetic breakdown is taken into account. Figure 13 shows a (001) cross section of the Fermi surface in the cubic-zone face. The cross-hatched area shows how a band of open orbits could propagate along [110] from one band to the other. If $t$ is the dimension over which there is an appreciable probability for breakdown near the point $T^{\prime}$, then the thickness of the band of open orbits is $t / \sqrt{2}$ when $\mathbf{B} \|[1 \overline{1} 0]$. Clearly, as $\mathbf{B}$ is rotated in the (001) plane away from [110] these open orbits will disappear. While this explanation gives the required open orbits along [110] it would also seem to provide the mechanism for open orbits along [001] when $\mathbf{B} \|[1 \overline{1} 0]$. Figure 14 shows a (110) cross section exhibiting [001] open orbits, also due to magnetic breakdown. There would be no coupling between this orbit and the lens orbit, because the relevant energy gap is $\sim 0.7 \mathrm{eV}$. If these orbits exist, we would again have two nonintersecting open orbits when $\mathbf{B} \|[110]$, and this would imply $m_{001} \rightarrow 0$ and $m_{110} \rightarrow 0$. The only conjecture we can make regarding this point is that, perhaps because of partial magnetic breakdown, the effective

${ }^{28}$ Roger Taylor, Bull. Am. Phys. Soc. 12, 22 (1967), and to be published.

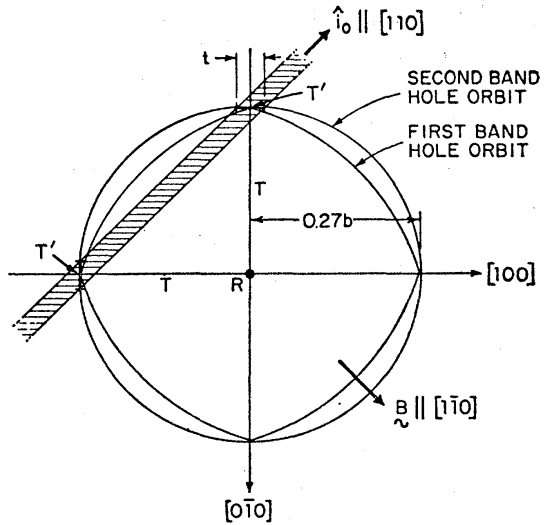

Fig. 13. Diagram showing a possible set of open orbits due to magnetic breakdown in [110] direction. $t$ is the dimension parallel to $\langle 100\rangle$ which measures the extent of the region of the magnetic breakdown (cross sections taken from Arlinghaus, Ref. 11).

open-orbit mobility of the [001] open orbits is significantly smaller than for the [110] orbits. In this connection, the dHvA signal due to the first-zone pockets vanishes when $\mathbf{B}$ is closer than about $6^{\circ}$ from $[1 \overline{1} 0]$ in the (110) plane ${ }^{4}$; this is consistent with the electrons tunneling from the first band to the second via magnetic breakdown. On the other hand, the amplitude of the dHvA signal coming from the second-band neck orbits is a maximum when $\mathbf{B} \|\langle 110\rangle .^{4}$ It is conceivable that this difference in behavior may be a result of inherently different relaxation times for the two pieces of Fermi surface; we note that the second-band orbit lies essentially in a $\{110\}$ zone face so that its wave function is likely to have a standing wave nature. Also, for those cyclotron orbits which lie precisely in the $\{110\}$ Brillouin zone faces, one would not expect magnetic breakdown to occur because the component of velocity normal to the zone face is zero; Eqs. (6) and (7) give a vanishing breakdown probability for this case.

There is other evidence for a small region of magnetic breakdown near the point $T^{\prime}$. As mentioned earlier,

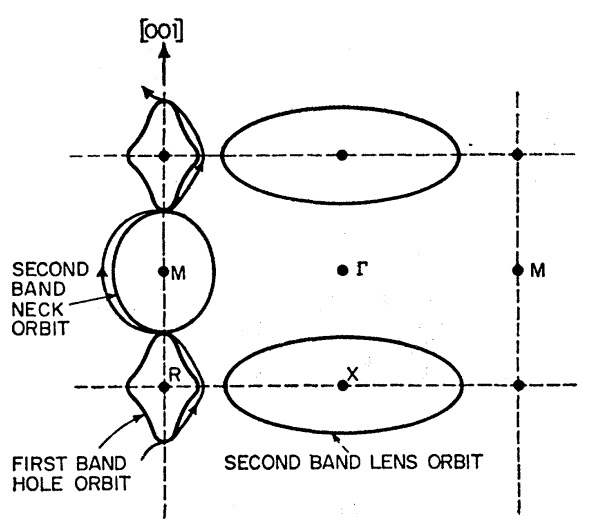

FIg. 14. Schematic diagram showing topology of a possible [001] open orbit due to magnetic breakdown. The plane of the section is (110) (not to scale). 
when $\mathbf{B} \|[001], m_{100} \rightarrow 1$ and $m_{110} \rightarrow 1$ (Table III). If there were no magnetic breakdown, symmetry alone would require that there exist no open or extended orbits when $\mathbf{B} \|[001]$ so that $m$ should approach zero unless we are not in the high-field region. This latter possibility does not seem likely, however, since for $\mathbf{B}$ in a nonsymmetry direction where there are no open orbits, $m \simeq 0.3$; if there are no open or extended orbits, the high-field region should be reached at essentially the same field value independent of the direction of $\mathbf{B}$, so that the linear behavior observed for $\mathbf{B} \|[001]$ is, in all probability, due to partial magnetic breakdown.

The behavior of the magnetoresistance when $\mathbf{B}$ is near $\langle 100\rangle$ is also suggestive of a small angular region of magnetic breakdown near the point $T^{\prime}$. Figures 6(a) and $6(\mathrm{~b})$ show that as the angle $\theta$ between $\langle 100\rangle$ and B becomes less than $\simeq 8^{\circ}$ the magnetoresistance increases sharply to a maximum at $\theta \simeq 4^{\circ}$ and then falls to a minimum at $\mathbf{B} \|\langle 100\rangle$ where $m \rightarrow 1$. The sharpness of the increase in the magnetoresistance at $\theta \simeq 8^{\circ}$ suggests that a new set of open orbits is opening up there; this interpretation is consistent with our earlier rough estimate of a magnetic-breakdown region of approximate radius $8^{\circ}$ around the lines $T$ ( $\langle 100\rangle$ directions).

Our final point is that if we had no Fermi-surface model to guide our interpretation, we would infer from the experimental results that a prominent feature of the Fermi surface is rather large "undulating" cylinders in the $\langle 110\rangle$ directions. The reasons for this are (1) the extent of the region of high magnetoresistance near [110] in Fig. 9, and (2) the magnitude of $\Delta \rho / \rho$ when B is close to $[1 \overline{1} 0]$. If we simply imagine cylindrical pieces of Fermi surface in the $\langle 110\rangle$ directions, then their diameter $d$ can be estimated from the width of the $[1 \overline{1} 0]$ shaded region of Fig. 9. The open orbits will be lost as $\mathbf{B}$ is tilted away from [110] toward [010] at an angle given by

\section{$\tan \theta \simeq d / b \sqrt{2}$.}

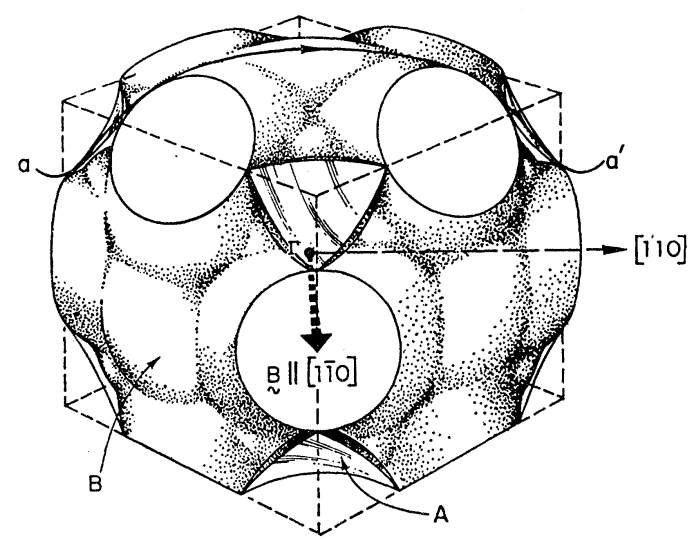

FIG. 15. Schematic diagram in extended-zone scheme showing open orbit $a a^{\prime}$ parallel to [110] when energy gaps on $\{100\}$ zone faces are broken through. $\mathbf{B}$ is parallel to [110] (out of page). The arrows point to pieces of Fermi surface in the first zone (A) and in the second zone (B).
Taking $\theta \simeq 21^{\circ}$ from the half-width of the [110] shaded region gives $d \simeq 0.5 b$; this can be regarded only as a rough estimate of $d$ because, as earlier work has shown, ${ }^{29}$ the angular dependence of the magnetoresistance at the edges of two-dimensional regions of open orbits depends critically on $\left\langle\omega_{c} \tau\right\rangle_{\mathrm{av}}$ as well as on specific geometrical details of the Fermi surface in question. Let us again consider our Fermi-surface model, in order to see how far the simple idea of undulating cylinders in the $\langle 110\rangle$ directions can be pushed. It is obvious from Fig. 15 that if we were to simply neglect the energy gap across the $\{100\}$ zone faces, we would easily have a large band of open orbits in the [110] direction; the thickness of the band would be given by the dimensions of the $\langle 110\rangle$ necks. If we take the dimensions of these necks in the $\langle 100\rangle$ and $\langle 110\rangle$ directions to be $d_{100}$ and $d_{110}$, respectively, then Arlinghaus gives $d_{100}=0.46 b$ and $d_{110}=0.42 b$, whereas Johnson and Amar give $d_{100}=0.58 b$ and $d_{110}=0.40 b$ (Table VI). Our estimate of $d \simeq 0.5 b$ should be compared with $d_{110}$ and, obviously, is in fair agreement with the theoretical resuls. While this hypothesis suggests an explanation of the data when $\mathbf{B}$ is near [110], it also requires that the $\{100\}$ energy gaps be so small (i.e., $\leqslant 0.1 \mathrm{eV}$ ) that magnetic breakdown can occur over a large part of the $\{100\}$ zone faces. That is, it does not seem possible for such a large region of open orbits to exist around $\langle 100\rangle$ if there is only a small angular region (e.g., of radius $8^{\circ}$ ) of magnetic breakdown around the lines $T$. There is no question that the $\{1 \overline{1} 0\}$ energy gaps are not identically zero as would be the case for disordered $\beta$-brass, because of the existence of $\mathrm{dHvA}$ oscillations (over largeangular regions) associated with the first-zone hole pockets centered on $R$.

\section{SUMMARY AND CONCLUSIONS}

The results of this research on ordered $\beta$-brass which can be stated with a fair degree of certainty are:

(1). It is an uncompensated metal in agreement with its odd number of electrons per unit cell.

(2). Its Fermi surface is multiply connected with open orbits existing when $\mathbf{B}$ lies in $\{100\}$ and $\{110\}$ planes; this is consistent with the predictions of existing energy-band calculations.

(3). It is necessary to invoke magnetic breakdown to explain the magnetoresistance when $\mathbf{B} \|\langle 100\rangle$ and $\mathbf{B} \|\langle 110\rangle$. The magnetoresistance results when $\mathbf{B}$ is near $\langle 110\rangle$ and parallel to $\langle 110\rangle$ do not submit to a simple explanation in terms of the theoretical model. We have given two possible explanations of the results; both of these involved eliminating one of the theoretically predicted open orbits, either by magnetic breakdown or by altering certain dimensions of the theoretical Fermi-

${ }^{29}$ See, for example, Ref. 17, N. E. Alekseevskii and Yu. P. Gaidukov, Zh. Eksperim. i Teor. Fiz. 42, 69 (1962) [English transl.: Soviet Phys.-JETP 15, 49 (1962)], and R. V. Coleman, A. J. Funes, J. S. Plaskett, and C. M. Tapp, Phys. Rev. 133, A521 (1964). 
surface model, and, in addition, required assumptions about various open orbits due to magnetic breakdown. With the latter of these explanations we were able to estimate a dimension of the $\langle 110\rangle$ necks which was in qualitative agreement with the model. However, because of the rather severe nature of the assumption about the magnitude of the $\{100\}$ energy gaps in this last explanation, it cannot be regarded as conclusive.

Finally, on the basis of the $\mathrm{dHvA}$ and magnetoresistance measurements, it seems clear that the energyband calculations are accurate in a semiquantitative way. $\beta$-brass is the first ordered alloy on which firstprinciples energy-band calculations and two types of Fermi-surface experiments have been done and the agreement is quite gratifying. The success obtained in ordered $\beta$-brass raises the hope that in the near future it will be possible to investigate quantitatively the electronic structure of more complicated ordered solids whose binding forces lie in the midst of the spectrum ranging from purely ionic to purely metallic.

\section{ACKNOWLEDGMENTS}

The authors at M.I.T. wish to acknowledge support of this work by the Advanced Research Projects Agency, through the Center for Materials Science and Engineering at M.I.T. We acknowledge the interest and support of Professor B. L. Averbach, and we thank P. J. Tobin and E. Sudenfield for help with some of the measurements. We are indebted to the staff of the National Magnet Laboratory at M.I.T. for the use of its facilities; in particular, L. G. Rubin was most helpful and cooperative. At the National Research Council, we thank A. Beck for skillfully preparing the specimens, Dr. R. Taylor for sending preliminary results prior to publication, Dr. P. T. Coleridge for reading the manuscript, and Dr. W. B. Pearson for his continued interest.

\title{
Quantum Oscillations in Helicon Attenuation
}

\author{
P. B. Miller and P. C. KwoK \\ IBM Watson Research Center, Yorktown Heights, New York
}

(Received 13 April 1967)

\begin{abstract}
The effect of the periodic potential on helicon attenuation is calculated using the effective-mass approximation. Above the Doppler-shifted cyclotron resonance large quantum oscillations arise from $\Delta n=0$ transitions which have nonvanishing matrix elements only for tilted orbits in real space. A comparison of the model with recent experiments on quantum oscillations in metals is discussed.
\end{abstract}

$T$ $\mathrm{HE}$ free-electron approximation in a magnetic field has been a convenient starting point for a discussion of the attenuation of helicon waves in metals. ${ }^{1-3}$ The additional effects of the periodic potential $\left(V_{p}\right)$ of the metal (i.e., real metal effects) on helicon attenuation may be separated into two categories: (a) a modification of electronic transitions which take place in the absence of $V_{p}$, and (b) the creation of new electronic transitions which do not occur in the absence of a periodic potential. Since the only transition which occurs in the absence of the periodic potential (when the magnetic field $H$ is parallel to the helicon wave vector $Q$ ) is $\Delta n= \pm 1$ ( $n=$ Landau quantum number), this transition may be described by the effect of the periodic potential on the corresponding absorption edge (i.e., on the Doppler-shifted cyclotron resonance ${ }^{1-5}$ ).

In this paper we discuss effects of type $b$ using the effective-mass approximation in a magnetic field. In

1 P. B. Miller and R. R. Haering, Phys. Rev. 128, 126 (1962).

2 P. M. Platzmann and S. J. Buchsbaum, Phys. Rev. 128, 1005 (1962).

${ }^{3}$ M. T. Taylor, J. R. Merrill, and R. Bowers, Phys. Letters 6 159 (1963); M. T. Taylor, Phys. Rev. 137, A1145 (1965).

${ }^{4}$ J. Kirsch and P. B. Miller, Phys. Rev. Letters 9, 421 (1962); J. Kirsch, Phys. Rev. 133, A1390 (1964).

5 E. A. Stern, Phys. Rev. Letters 10, 91 (1963). the magnetic field region above the Doppler-shifted absorption edge $\left[H>H_{D}\right]$, the energy separation between Landau levels is so large that the real electronic transitions are limited by energy-momentum conservation to $\Delta n=0$ and $\Delta k_{z}=Q$, where $\hbar k_{z}$ is the electronic momentum along the field. In a geometry where the magnetic field is parallel to the helicon wave vector $Q$, the matrix element for the $\Delta n=0$ transition vanishes in the free-electron approximation. Therefore, in this geometry a clear separation of real metal effects from free-electron effects occurs, i.e., above the Dopplershifted absorption edge all attenuation in a pure metal must be due to real metal effects.

Quantum oscillations in helicon attenuation have been observed for $H>H_{D}$ in several metals by Grimes ${ }^{6}$ and by Grimes and Libchaber. ${ }^{7}$ A theoretical model of helicon attenuation based on the effective-mass approximation shows that the main effect of the periodic potential is to produce a "tilted orbit" effect in real space. The "tilted orbit" effect leads to a qualitative

${ }^{6}$ C. C. Grimes, in Proceedings of the Symposium on Plasma Effects in Solids, Paris, 1964 (Dunod, Cie., Paris, 1964).

${ }_{7}$ C. C. Grimes and A. Libchaber (to be published) and private communication. 